\title{
Immunomodulatory effect of Bifidobacterium breve on experimental allergic rhinitis in BALB/c mice
}

\author{
JIANJUN REN ${ }^{1 *}$, YU ZHAO $^{1}$, SHI HUANG $^{2 *}$, DAN LV $^{1}$, FENGLING YANG $^{1}$, LIN LOU $^{1}$, \\ YONGBO ZHENG ${ }^{1}$, JIE ZHANG ${ }^{3}$, SHIXI LIU ${ }^{3}$, NAN ZHANG ${ }^{4}$ and CLAUS BACHERT ${ }^{4}$ \\ ${ }^{1}$ Department of Otorhinolaryngology, West China Hospital, West China Medical School, Sichuan University, Chengdu, \\ Sichuan 610041; ${ }^{2}$ Department of Otorhinolaryngology, Head and Neck Surgery, The First Affiliated Hospital of \\ Chengdu Medical College, Chengdu, Sichuan 610000; ${ }^{3}$ Upper Airways Research Laboratory, \\ West China Hospital, Sichuan University, Chengdu, Sichuan 610041, P.R. China; \\ ${ }^{4}$ Upper Airways Research Laboratory, Department of Otorhinolaryngology, Ghent University, 9000 Ghent, Belgium
}

Received December 19, 2017; Accepted May 17, 2018

DOI: $10.3892 /$ etm.2018.6704

\begin{abstract}
Bifidobacteriumbreve(B.breve) may have abeneficial effect on allergic rhinitis (AR). The aim of the present study was to investigate whether microbial induction of regulatory $\mathrm{T}$ cells (Tregs) and adjustment of Th1 and Th2 responses by $B$. breve are associated with protection against allergic inflammation, and to identify a dose-response association in a murine AR model. Ovalbumin (OVA)-sensitized BALB/c mice were orally treated with different doses of $B$. breve $\left[10^{10}, 10^{9}, 10^{7}\right.$ and $10^{5}$ colony forming units (CFU)]. Following nasal challenge with OVA, sneeze frequency, serum OVA-specific immunoglobulin $\mathrm{E}$ (IgE) and cytokine concentrations [interleukin (IL)-4, IL-10, IL-13 and interferon- $\gamma$ ], splenic percentage of cluster of differentiation (CD)4+CD25+ Tregs, and morphology of the nasal mucosa were examined. Oral treatment with live $B$. breve at doses of $10^{7} \mathrm{CFU}$ or higher alleviated nasal mucosal injury and suppressed sneezing upon repeated administration over a 6 -week period. Furthermore, treatment with $B$. breve at these higher doses reduced the concentrations of serum OVA-specific IgE, IL-4 and IL-10, and increased the splenic percentage of CD4+CD25+ Tregs in rhinitic mice compared with those who did not receive probiotics. In contrast, treatment with $B$. breve at a lower dose did not indicate any effect on sneezing frequency or mucosal morphology in this animal model, even though the splenic percentage of $\mathrm{CD} 4+\mathrm{CD} 25+$ Tregs increased and the concentrations of serum OVA-specific IgE and IL-10 declined.
\end{abstract}

Correspondence to: Professor Yu Zhao, Department of Otorhinolaryngology, West China Hospital, West China Medical School, Sichuan University, 37 Guo Xue Alley, Chengdu, Sichuan 610041, P.R. China

E-mail: yutzhao@163.com

${ }^{*}$ Contributed equally

Key words: allergic rhinitis, Bifidobacterium breve, probiotics, regulatory $\mathrm{T}$ cells
B. breve exerts its anti-allergic effects by inhibiting type 2 helper $\mathrm{T}$ cell immune responses and enhancing CD4+CD25+ Treg activity. Sneezing was also reduced at a dose of $10^{7} \mathrm{CFU}$ or higher. The current study investigated the role of $B$. breve and aided in identifying the optimal dose of $B$. breve administration in the treatment of AR.

\section{Introduction}

In recent years, allergic rhinitis (AR) has emerged as a major public health problem worldwide. To date, the underlying immunological mechanisms of AR are not fully understood, as it is a complex multifactorial disorder, involving both innate and adaptive immune responses. AR is classified in the category of type I allergic diseases, which are generally characterized by an elevation in serum immunoglobulin E (IgE) levels (1). This is mediated mainly by type 2 helper T (Th2) cells. These cells synthesize high levels of interleukin (IL)-4, IL-5 and IL-13, which lead to the production of allergen-specific $\mathrm{IgE}$ and the release of histamine and leukotriene from mast cells (2). Conversely, type 1 helper T (Th1) cells suppress Th2 immune responses by secreting interferon- $\gamma($ IFN- $\gamma)(3)$. As the influx and differentiation of Th2 cells are important factors in the development and aggravation of AR, previous research has focused on the activation of Th2 cells or modulation of the Th1/Th2 balance to prevent and treat AR: A number of previous studies have investigated the role of activation of regulatory $\mathrm{T}$ cells (Tregs) or the induction of immunologic tolerance in the development of allergic diseases $(4,5)$. Tregs can inhibit the differentiation of Th0, their common precursor, intoTh2 cells $(6,7)$. Therefore, Tregs may serve an important role in the pathogenesis of AR, controlling immune responses to self and nonself antigens (8). IL-10 is a general inhibitor of proliferative and cytokine responses in $\mathrm{T}$ cells, is produced by mononuclear phagocytes, natural killer cells and Th1 and Th2 type lymphocytes (9) and may be the key factor in the immunoregulation.

Probiotic bacteria are non-invasive and non-pathogenic living microorganisms, which typically colonize in the intestinal and reproductive system, and, when consumed in adequate numbers, may confer certain health benefits 
on the host. An ameliorating effect has been reported on a range of human diseases including infectious diseases (10), gastrointestinal disorders (11) and allergic diseases (12), and the immunomodulating activity has been demonstrated as an important effect (13). Recent reports $(14,15)$ have documented the role of probiotics in the prevention of allergic disorders, and it is increasingly recognized that probiotics serve a central role not only in Th1/Th2 polarization, but also in inducing the appropriate regulatory mechanisms. Although the specific mechanism is still unclear, reduced serum levels of ovalbumin (OVA)-specific IgE may be associated with enhanced production of Th1 cytokines, decreased synthesis of Th2 cytokines, or both (16). Other reports have identified strain-dependent differences in the ability of probiotics to modulate immune responses. By comparing the anti-allergic treatment effects of four strains of Bifidobacteria and 2 strains of Lactobacilli, Hougee et al (17) demonstrated that oral treatment with $B$. breve was most effective in reducing allergic responses. The present authors previously compared the anti-allergic qualities of $B$. breve, Lactobacillus and mixed probiotic (B. breve and Lactobacillus) species by analyzing Th1 and Th2 cytokine levels and the splenic percentage of cluster of differentiation (CD) $4^{+} \mathrm{CD} 25^{+}$Tregs, and confirmed $B$. breve as the bacterial strain with the most potent anti-allergic treatment effects (unpublished data). In addition, it has been demonstrated that oral treatment with probiotics at doses of $10^{7}-10^{10}$ colony forming units (CFU) suppresses serum antigen-specific $\mathrm{IgE}$ in animal models $(8,18)$. However, there are also reports from both in vitro and clinical studies indicating that a lower dose of $B$. breve is more effective than higher doses in the treatment of allergic diseases $(19,20)$. Due to these conflicting results, the optimal dose of oral administration of $B$. breve remains to be determined.

The aim of the present study was to investigate the mechanisms associated with the anti-allergic effects of $B$. breve in the treatment of AR, and to identify the optimal dose of $B$. breve administration.

\section{Materials and methods}

Animals. A total of 60 female BALB/c mice (age, 6-8 weeks; weight, 20-25 g) purchased from Chengdu Dashuo Laboratory Animal Co, Ltd (Chengdu, China) were acclimatized to the laboratory environment for 1 week prior to the beginning of the experiment. Mice were housed in type III Macrolon cages and provided with standard rodent food and tap water ad libitum. Temperature and humidity were kept constant at $24 \pm 1^{\circ} \mathrm{C}$ and $50 \%$, respectively, with a 12 -h light/dark cycle under specific-pathogen-free condition (21). All animals were randomly distributed to 6 treatment groups ( $n=10$ per group; Table I), and all animal experiments were performed according to the Guide for the Care and Use of Laboratory Animals (22) and approved by the Laboratory Animal Ethics Committee of Sichuan University (Chengdu, China).

OVA sensitization and challenge. Mice were sensitized on Day 0 and Day 7 by intraperitoneal injections with phosphate-buffered saline (PBS) or $20 \mu \mathrm{g}$ OVA grade V (Sigma-Aldrich; Merck $\mathrm{KGaA}$, Darmstadt, Germany) in $2 \mathrm{mg} / 0.1 \mathrm{ml} \mathrm{Al}(\mathrm{OH})_{3}$ gel (Thermo Fisher Scientific, Inc., Waltham, MA, USA) suspended in PBS. Nasal challenge was performed by topical application of PBS or $20 \mu \mathrm{l}$ OVA solution $(10 \mathrm{mg} / \mathrm{ml})$ via nasal drops once daily from Day 14-56. A schematic overview of the study design is presented in Table I and Fig. 1.

Probiotic treatment. The B. breve strain was obtained from the Institute of Biology and Pharmacology of Shanghai Jiao Tong University (Shanghai, China). Live bacteria powder was suspended in $0.2 \mathrm{ml}$ PBS ( $\mathrm{pH} 7.2$ ) at concentrations of $10^{10}$, $10^{9}, 10^{7}$ and $10^{5} \mathrm{CFU}$ prior to administration. B. breve (or PBS) was administered by oral gavage once daily from Day 14 until the mice were sacrificed on Day 56.

Observation of nasal symptoms. Mice were placed in an observation cage, to allow counting of the total number of sneezes by reviewing video records of the animals' daily activities. Counting commenced immediately following the final intranasal challenge, for a period of $10 \mathrm{~min}$.

Serum OVA-specific IgE levels. ELISA was performed on serum samples to detect IgE levels according to the manufacturer's instructions (439807; BioLegend, Inc., San Diego, CA, USA). Samples were collected following a nasal stimulation test as described and were allowed to clot for $\geq 30 \mathrm{~min}$ prior to centrifugation for $10 \mathrm{~min}$ at $1,000 \mathrm{x} \mathrm{g}\left(2-8^{\circ} \mathrm{C}\right)$. Serum layers were collected separately and stored at $-80^{\circ} \mathrm{C}$ prior to analysis. Serum samples were diluted 1:1-1:6 in standard stock solution to meet the kits standard testing ranges. Absorbance was measured at $450 \mathrm{~nm}$ on a Bio-Rad 680 microplate reader (Bio-Rad Laboratories, Inc., Hercules, CA, USA). Total immunoglobulin levels were calculated with reference to a standard curve using the following equation: Total Ig level=ELISA value (A450) $\mathrm{x}$ dilution factor, where $\mathrm{A}$ is absorbance. The minimal detection concentration for OVA-specific IgE was $20.7 \mathrm{pg} / \mathrm{ml}$.

Cytokine levels. IL-4, IL-10, IL-13 and IFN- $\gamma$ levels in serum were determined with Luminex kits (LUM000; R\&D Systems, Inc., Minneapolis, MN, USA) according to the manufacturer's instructions and analyzed using a Luminex100 instrument. Samples were diluted 1:2 in standard stock solution.

Histopathological analysis. Mice were sacrificed on Day 56 to collect nasal tissue for histological observation. The head was removed and the lower jaw, skin and soft tissue were discarded. The skull was decalcified in $10 \%$ nitric acid solution for 5 days prior to $10 \%$ formalin fixing for 2 days at room temperature and paraffin embedding $\left(12 \mathrm{~h}, 60^{\circ} \mathrm{C}\right)$. Serial sections $(4-\mu \mathrm{m})$ were cut at the level of middle turbinate and inferior turbinate and stained with hematoxylin for $1-3 \mathrm{~min}$ at $25^{\circ} \mathrm{C}$ and eosin $30-60 \mathrm{sec}$ at $25^{\circ} \mathrm{C}$ for the detection of infiltrating eosinophils. Sections were reviewed by light microscopy at a magnification, $\mathrm{x} 400$.

Splenic Treg analysis. The spleen was removed and placed on ice, and half of the organ was gently ground prior to filtering through a $40-\mu \mathrm{m}$ filter to obtain cell suspensions, as detailed previously (7). An eBioscience ${ }^{\mathrm{TM}}$ Mouse Regulatory T Cell Staining kit (88-8115; eBioscience; Thermo Fisher Scientific, Inc.) was used according to the manufacturer's instructions. Approximately $10^{6}$ splenocytes suspended in $1 \mathrm{ml}$ PBS were stained with fluorescein isothiocyanate-labeled anti-mouse 
Table I. Grouping information and probiotics treatment.

\begin{tabular}{|c|c|c|c|c|c|c|}
\hline Criteria & Negative control group & Positive control group & $10^{10}$ group & $10^{9}$ group & $10^{7}$ group & $10^{5}$ group \\
\hline Number of animals & 10 & 10 & 10 & 10 & 10 & 10 \\
\hline Sensitization & PBS & $20 \mu \mathrm{g}$ OVA & $20 \mu \mathrm{g}$ OVA & $20 \mu \mathrm{g}$ OVA & $20 \mu \mathrm{g}$ OVA & $20 \mu \mathrm{g}$ OVA \\
\hline Nasal challenge & PBS & $200 \mu \mathrm{g}$ OVA & $200 \mu \mathrm{g}$ OVA & $200 \mu \mathrm{g}$ OVA & $200 \mu \mathrm{g}$ OVA & $200 \mu \mathrm{g} \mathrm{OVA}$ \\
\hline Probiotic dose & PBS & PBS & $10^{10} \mathrm{CFU}$ & $10^{9} \mathrm{CFU}$ & $10^{7} \mathrm{CFU}$ & $10^{5} \mathrm{CFU}$ \\
\hline
\end{tabular}

OVA, ovalbumin; CFU, colony forming units.

A

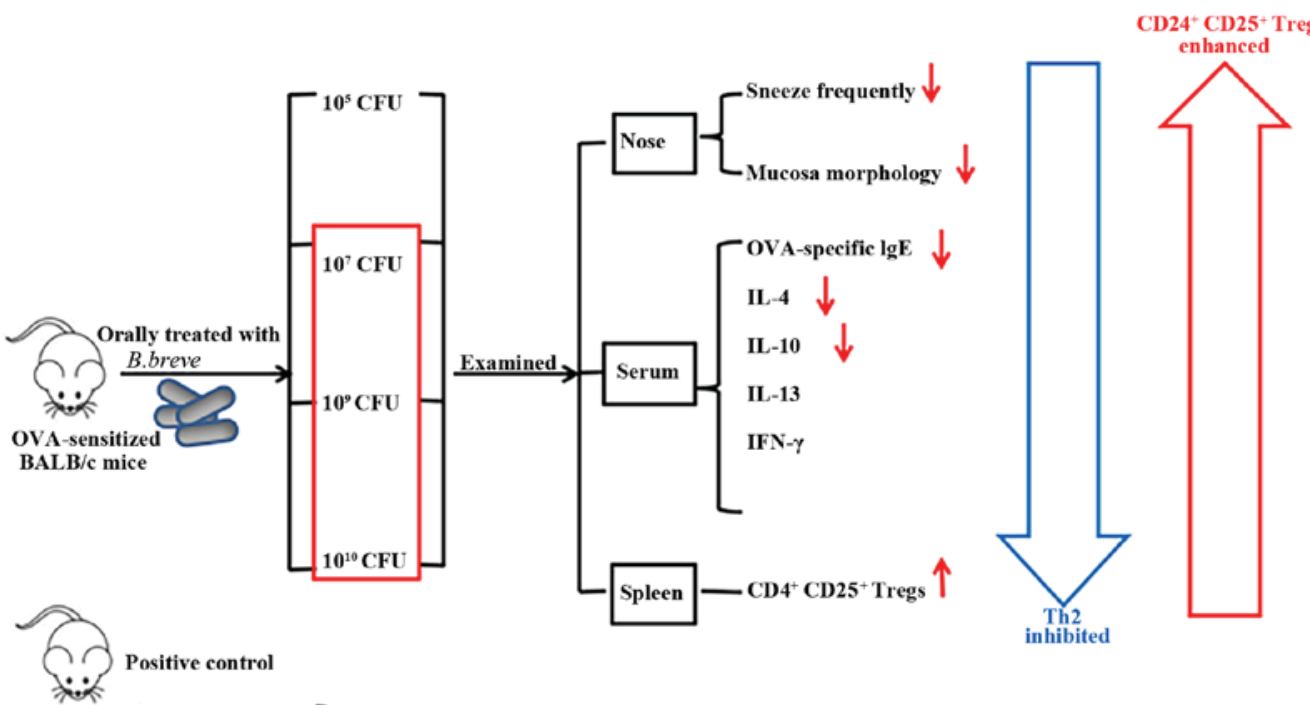

(AR model without $B$ breve treatment)

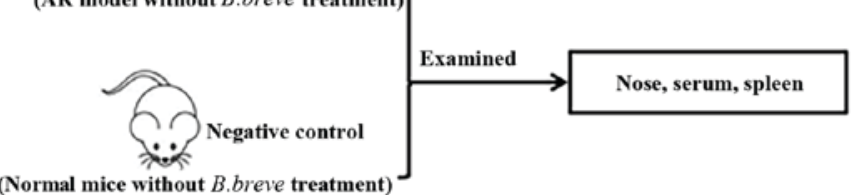

(Normal mice without B.breve treatment)

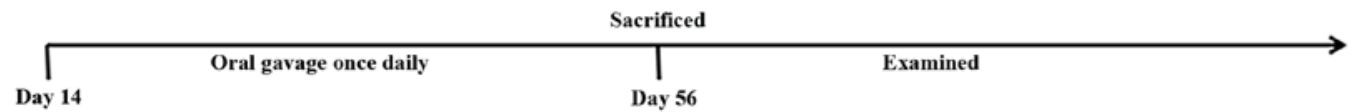

B

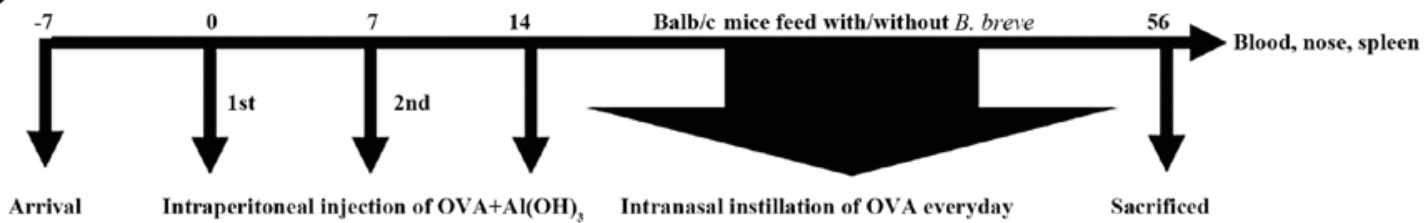

Time (days)

Daily probiotic feeding

Figure 1. Study design. (A) Graphic abstract for the present study. (B) The experimental protocol. Mice were given probiotics daily from Day 0-56, with OVA-sensitization on Days 0 and 7, and nasal challenge with OVA from Day 14-56. Mice were sacrificed on Day 56 to collect tissue for experimental analysis. OVA, ovalbumin; CFU, colony forming units; IgE, immunoglobulin E; IL, interleukin; IFN, interferon; CD, cluster of differentiation; AR, allergic rhinitis.

CD4 $(0.625 \mu \mathrm{g} / \mathrm{ml})$ and phycoerythrin-labeled anti-mouse CD25 antibodies $(0.3 \mu \mathrm{g} / \mathrm{ml})$ provided with the kit for $30 \mathrm{~min}$ at $4^{\circ} \mathrm{C}$. According to the manufacture's instructions permeabilization and fixation $\left(30 \mathrm{~min}\right.$ at $4^{\circ} \mathrm{C}$ ) was performed prior to blocking with anti-mouse CD16/CD32 purified antibody for $15 \mathrm{~min}$ at room temperature. The splenocytes were intracellularly stained with APC-labelled anti-mouse forkhead box P3 (Foxp3) antibodies $(2.5 \mu \mathrm{g} / \mathrm{ml}$, provided with the kit) and incubated for $30 \mathrm{~min}$ at room temperature. Treg cells were detected by flow cytometry (BD, FACSAria ${ }^{\mathrm{TM}}$, Biosciences, Franklin Lakes, NJ, USA) and data were analyzed with FlowJo 7.6 software (Tree Star, Inc., Ashland, OR, USA). 
Statistical analysis. Statistical analysis was performed using SPSS 17.0 (SPSS, Inc., Chicago, IL, USA). Data were expressed as the mean \pm standard error of the mean. Normal distribution and homogeneity of variance were tested with Q-Q plotting and Levene's Test, respectively. For data with normal distribution and homogeneity of variance, one-way analysis of variance was applied to analyze the differences among multiple groups, followed by Student-Newman-Keuls or Dunnett's post hoc tests. For data without normal distribution, a non-parametric Kruskal-Wallis test was applied to test whether there was a significant difference between the multiple distributions. If so, a Nemenyi test was conducted for further comparison. $\mathrm{P}<0.05$ was considered to indicate a statistically significant difference.

\section{Results}

Sneeze frequency. The number of sneezes was recorded on Day 56 during a 10 -min period starting immediately following the last nasal challenge. As presented in Fig. 2, the sneeze frequency was lower in the negative control group, compared with the positive control group. Oral treatment with $B$. breve at doses of $10^{10}, 10^{9}$ and $10^{7} \mathrm{CFU}$ significantly reduced the number of sneezes compared with the positive control group, without significant differences among these 3 treatment groups. Treatment with $10^{5} \mathrm{CFU}$ did not have any significant effect on sneezing frequency in comparison with the positive control group.

Serum OVA-specific IgE. Levels of OVA-specific IgE were significantly lower in the negative control group compared with the positive control group, indicating that the OVA sensitization was successful. Oral treatment with all four doses of $B$. breve significantly reduced OVA-specific IgE, in comparison with the positive control group (Fig. 3).

Impact of B. breve on serum cytokine levels. IL-4 and IL-10 levels were lower in the negative control group and all probiotic treatment groups compared with the positive control group. This reduction was significant for all probiotic groups except for the $10^{5}$ group in terms of IL-4 levels. There were no significant differences between the $10^{10}, 10^{9}$ and $10^{7}$ groups. There were no significant differences among the 6 groups in terms of IL-13 and IFN- $\gamma$ levels (Fig. 4).

Splenic Treg cells. The percentage of $\mathrm{CD} 4{ }^{+} \mathrm{CD} 25^{+}$Tregs was significantly lower in the positive control group compared with the negative control group. All tested doses of $B$. breve caused a significant increase in the percentage of CD $4^{+} \mathrm{CD} 25^{+}$Tregs, compared with the positive control group. However, there were no significant differences between different dose groups (Fig. 5). Interestingly, CD $4^{+} \mathrm{CD} 25^{+}$Foxp 3 Tregs demonstrated no significant difference between groups following repeating experiment several times (data not shown), the reasoning for which was unclear. Therefore, only $\mathrm{CD} 4^{+} \mathrm{CD} 25^{+}$Tregs were used.

Effects of B. breve treatment on nasal mucosal inflammation. The nasal mucosal epithelium was intact following treatment with $B$. breve at doses of $10^{10}, 10^{9}$ and $10^{7} \mathrm{CFU}$, and nasal

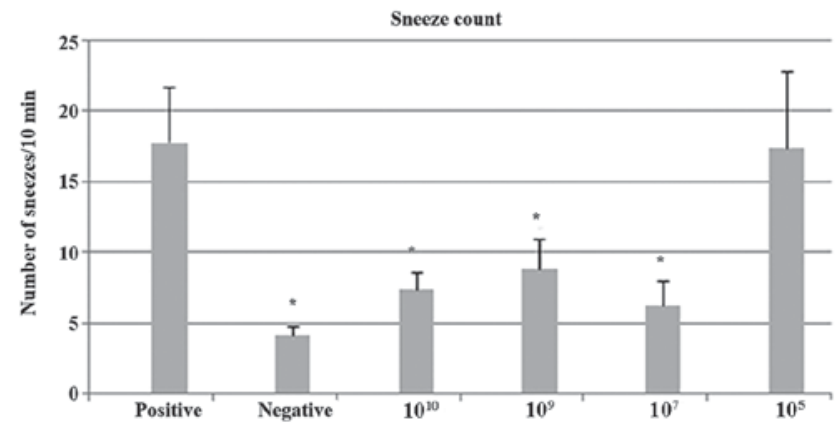

Figure 2. Sneeze counts in control groups and B. breve administered groups The sneeze frequency was lower in the negative control group and groups treated with $B$. breve at doses of $10^{10}, 10^{9}$ and $10^{7} \mathrm{CFU}$ compared with the positive control. Treatment with 105CFU did not have any significant effect on sneezing frequency. ${ }^{*} \mathrm{P}<0.05$ vs. positive. $\mathrm{CFU}$, colony forming units.

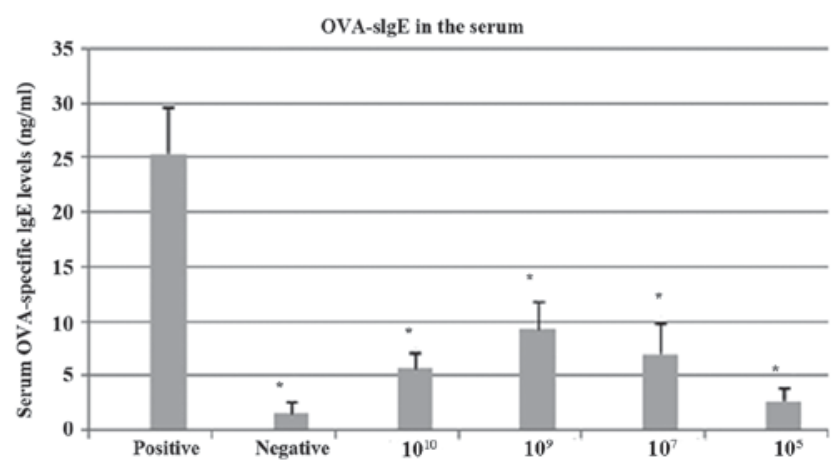

Figure 3. Serum OVA-specific IgE levels detected in each group. Serum OVA-specific IgE was significantly lower in the negative control group compared with the positive control group. Oral treatment with all four doses of $B$. breve significantly reduced OVA-specific IgE compared with the positive control group. ${ }^{*} \mathrm{P}<0.05$ vs. positive. OVA, ovalbumin; IgE, immunoglobulin E.

mucosal congestion was reduced compared with the positive control group; however, the $10^{5}$ group did not markedly alleviate nasal mucosal edema (Fig. 6).

\section{Discussion}

AR evokes an excessive Th2 reaction, leading to high levels of IL-4, IL-5 and IL-13 in serum (2). In the present study, a significant increase of OVA-specific IgE level was detected following OVA sensitization in the positive control group compared with the negative control group, confirming the establishment of sensitization. The present findings, that probiotics are capable of inhibiting OVA-specific IgE production when administered orally following sensitization, are supported by the available literature $(23,24)$. The present results suggest that $B$. breve intervention reduces the production of OVA-specific IgE and can relieve type I allergy disease in mice. Administration of $B$. breve at doses of $10^{10}, 10^{9}$ and $10^{7}$ CFU significantly decreases the OVA-specific IgE levels and sneezing frequency, and nasal mucosal epithelium was protected from local allergic reaction. $B$. breve administration with $10^{5} \mathrm{CFU}$ significantly decreased the OVA-specific IgE levels, whereas sneezing frequency and nasal mucosal damage were unchanged compared with the positive control 
A

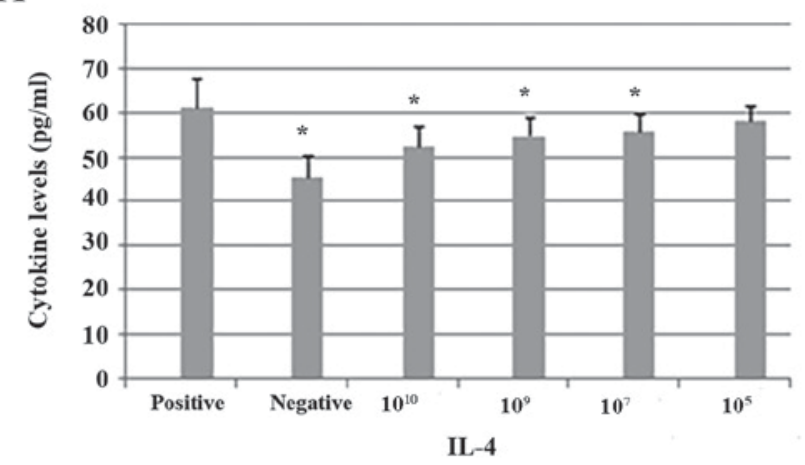

C

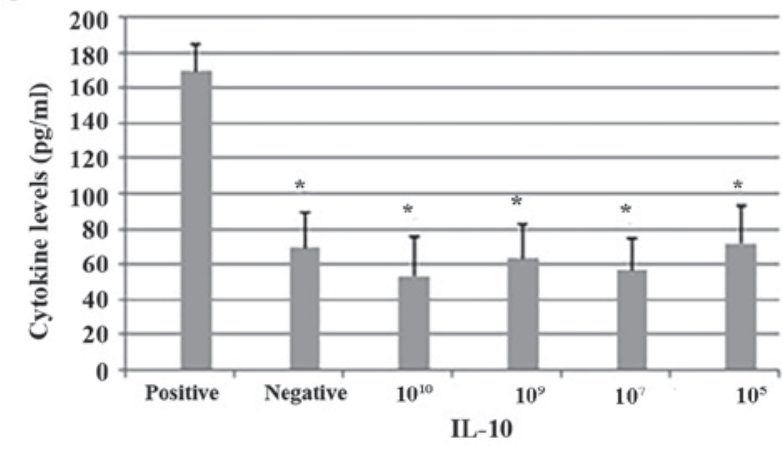

B

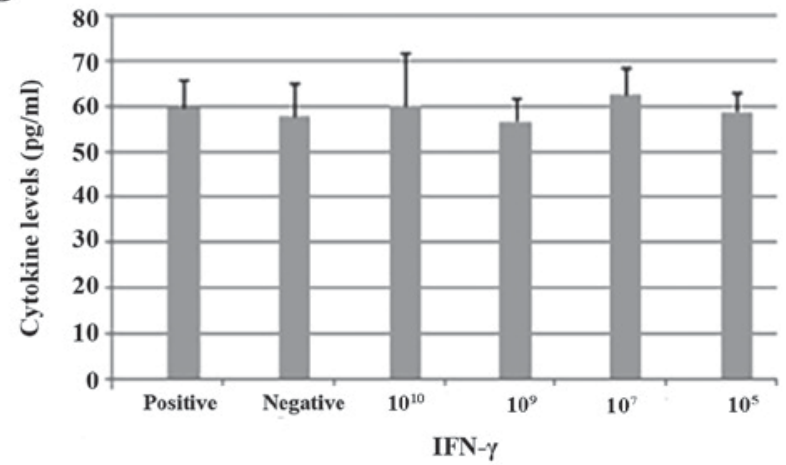

D

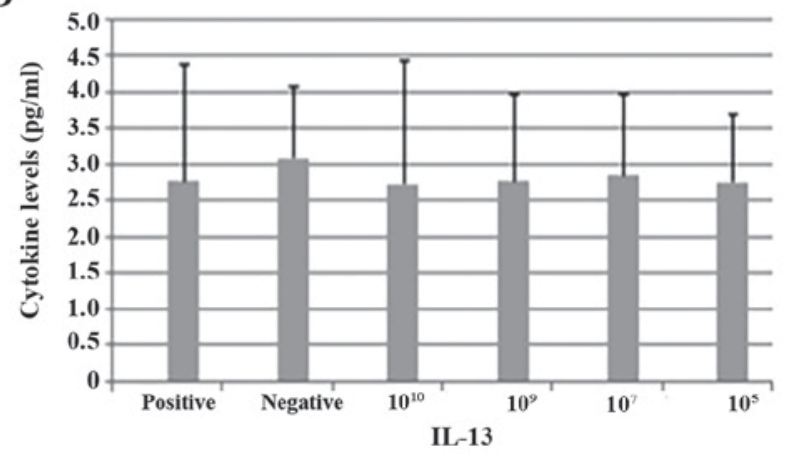

Figure 4. Cytokine levels in OVA-sensitized mice following repeated administration of different doses of $B$. breve. (A) IL-4, (B) IFN- $\gamma$, (C) IL-10 and (D) IL-13 levels were measured. IL-4 and IL-10 levels were significantly lower in the negative control group and all probiotic treatment groups compared with the positive control group, with the exception of the 105 group in terms of IL-4 levels. There were no significant differences between the $10^{10}$, $10^{9}$ and $10^{7}$ groups. No differences among the six groups in terms of IL-13 and IFN- $\gamma$ levels were observed. * $\mathrm{P}<0.05$ vs. positive. OVA, ovalbumin; IL, interleukin; IFN, interferon.

group, indicating that this lower dose of $B$. breve only slightly relieved the allergic reaction.

In the intestinal immune system, Th1 and Th2 cells influence the production of IgE. Th2 cells secrete IL-5, IL-13 and IL-4, the characteristic factors leading to enhanced $\operatorname{IgE}$ production, eosinophilia and atopic disease (25). Several studies have suggested that Th2-cytokines, particularly IL-4 and IL-13, serve a pivotal role in the pathogenesis of allergic diseases by regulating the production of $\operatorname{IgE}$ and activation of mast cells (26-28). In contrast, Th1 cells secrete IL-2, IL-12 and IFN- $\gamma$, which are responsible for suppression of IgE synthesis as well as the inception of IgE-mediated allergies (29). AR has often been reported to be associated with defective IFN- $\gamma$ expression (30). The immunoregulatory effects of probiotics have been ascribed to shifting the Th1/Th2 balance towards Th1 mediated immunity, which might inhibit the development of allergies (31), as was confirmed by $\operatorname{IgE}$ suppression by $B$. breve in animal allergy models. Despite clear evidence for strong immunomodulatory properties of $B$. breve, the mechanisms underlying these effects are poorly understood. The associated cytokine patterns in OVA-stimulated splenocytes from Bifidobacterium-fed mice were highly strain dependent, which indicates that different mechanisms are involved. Reduced serum levels of OVA-specific IgE may be associated with enhanced production of Th1 cytokines, decreased synthesis of Th 2 cytokines, or both in some instances, as both Th1 and Th2 cytokines were down regulated (16). The present study demonstrated that IL-4 secretion was significantly lower in the $10^{10}, 10^{9}$ and $10^{7} \mathrm{CFU}$ probiotic groups than in the positive control group, supporting a reduced Th2 response, whereas there was no difference in Th1 representative factors IFN- $\gamma$. This suggests that $B$. breve instates Th1/Th2 equilibrium mainly through the inhibition of the Th2 response, without promoting a Th1 response. Previous studies have indicated that the immunoregulatory mechanism of oral probiotics depends on the specific bacterial strain used $(32,33)$. Research on $B$. breve administration demonstrated conflicting results concerning both the mechanisms and treatment effects, and the differences in outcome may be attributed to the use of different probiotic doses of probiotics $(17,18)$. Four different doses of $B$. breve were administered to mice by oral gavage. It was speculated that while different doses of probiotics may indicate varied immunoregulatory capacity, the underlying mechanism is the same.

The spleen is the organ where lymphocytes accept antigen stimulation like the lymph nodes, and give rise to specific immune responses. Tregs are the mediators of immunological tolerance and possess anti-inflammatory capabilities (34). They comprise a diverse group of cells, including CD4+CD25+ $\mathrm{T}$ cells, which can suppress antigen-specific responses even in small numbers. Abnormalities in number and function may cause autoimmune diseases (35). In the present study, the ratio of CD4+CD25+ T cells was significantly reduced in the positive control group compared with the negative control group. As CD4+CD25+ T cells can inhibit the differentiation of Th0 cells into Th2 type and the fact that IL-10 is required in the development of Tregs $(6,7)$, it was speculated that inhibition of nasal symptoms by $B$. breve administration may be associated with the induced activity of Tregs. Tolerogenic dendritic cells may be the source of IL-10 for the induction of Tregs (8); therefore, 
$\mathbf{A}$
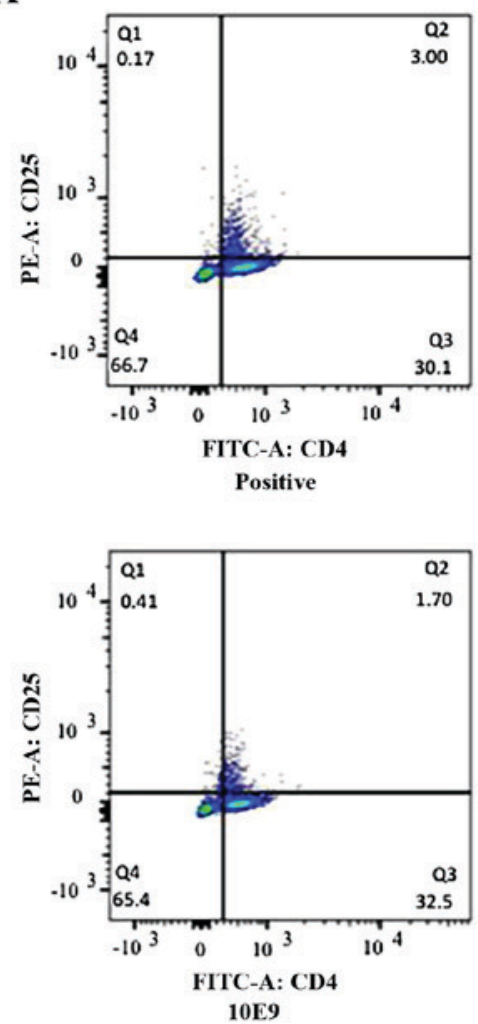

B

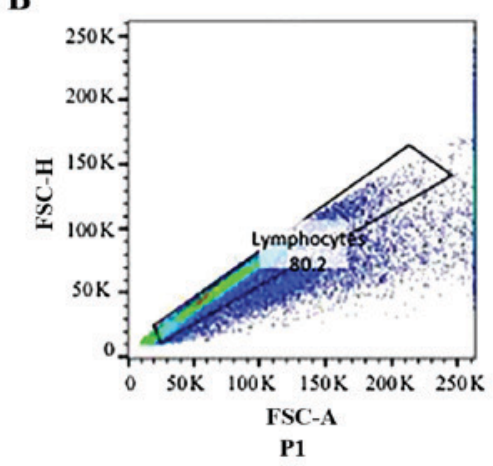

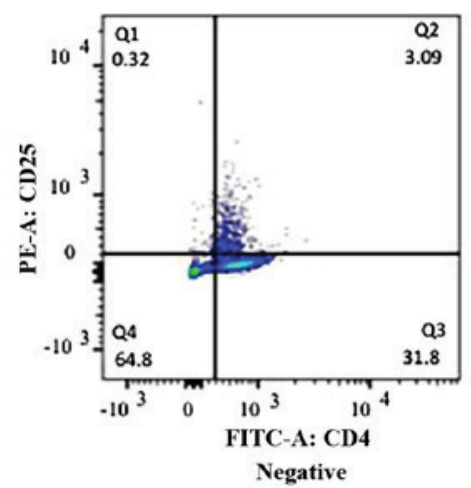

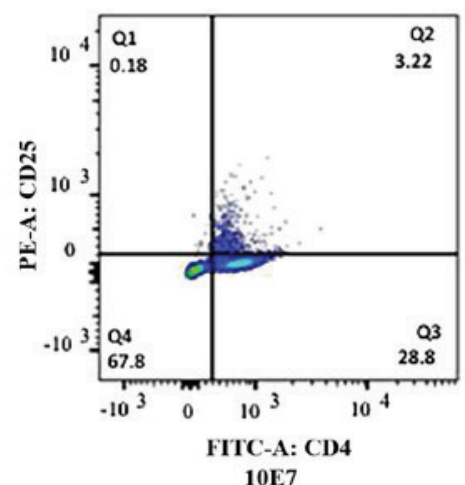

$10 \mathrm{E} 7$

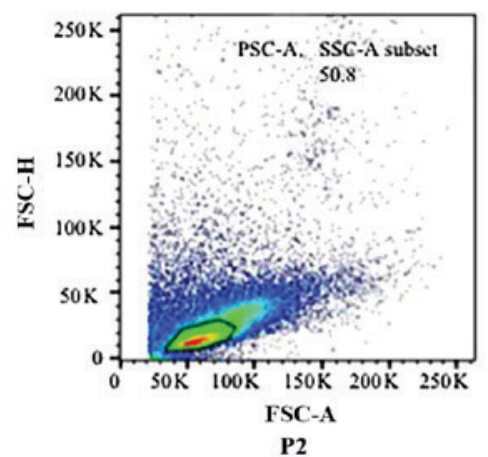

P2
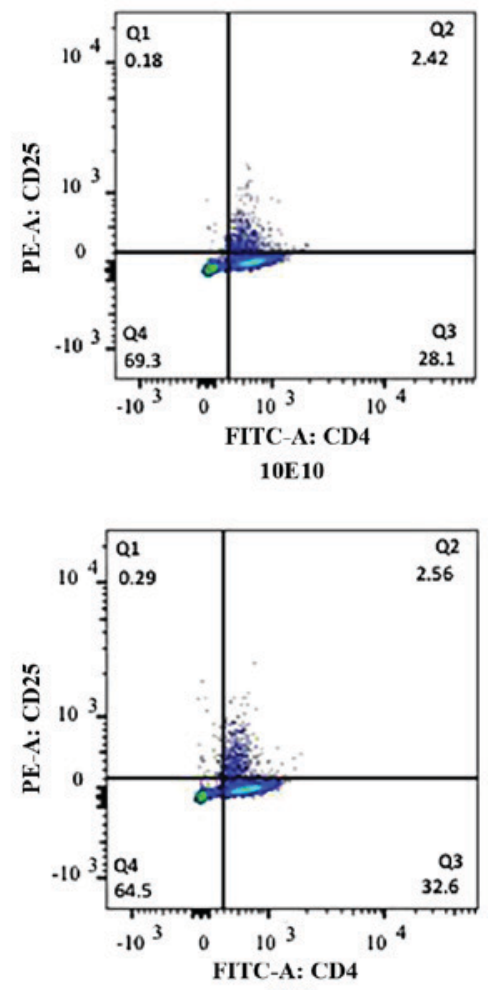

$10 \mathrm{E} 5$

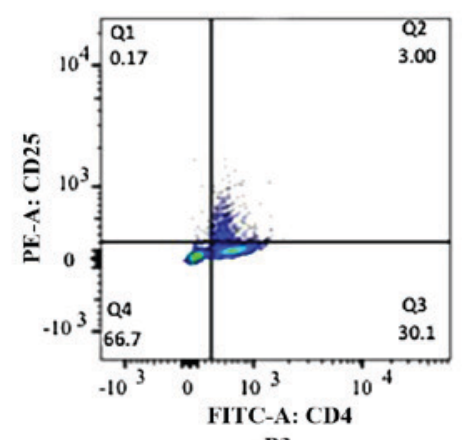

P3

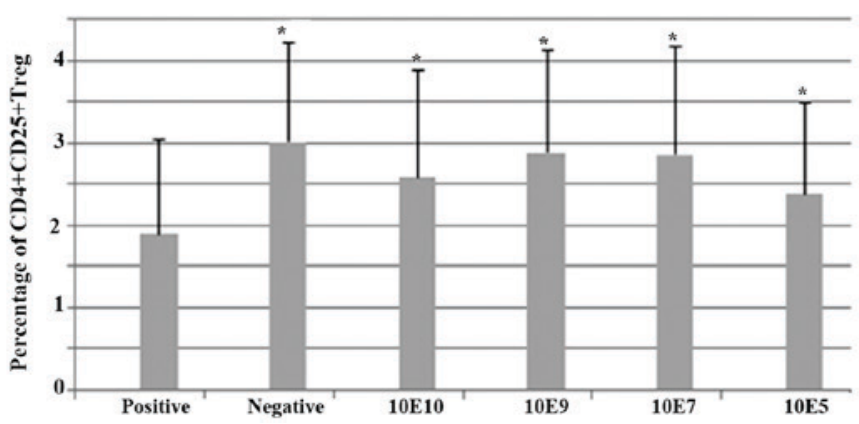

Figure 5. Percentage of CD4+CD25+ Tregs in the spleen. (A) Representative flow cytometry figures of CD4 and CD25 staining of splenocytes from 1 mouse in each treatment group and control group. Cells were stained with fluorescent-labeled anti-CD4 and -CD25 antibodies and analyzed in a flow cytometer. (B) Gating strategy for flow cytometry analysis. In this sample gating, cells were first gated for singlets (P1: FSC-H vs. FSC-A) and lymphocytes (P2: SSC-A vs. FSC-A). The lymphocyte gate was further analyzed for identifying CD25 and CD4, which were stained with FITC and PE, respectively (P3: PE-CD25 vs. FITC-CD4). (C) Percentage of splenic CD4+CD25+Tregs among six groups. " $\mathrm{P}<0.05$ vs. positive. $\mathrm{CD}$, cluster of differentiation; Treg, regulatory T cell; FSC-H, forward scatter height; FSC-A, forward scatter area; SSC-A, side scatter area; Q2, CD4+CD25+Tregs; FITC, fluorescein isothiocyanate; PE, phycoerythrin.

it has been proposed that probiotics promote the production of IL-10 to induce Tregs. In this regard, a number of studies have confirmed that the symptoms of colitis are significantly relieved by probiotic treatment in IL-10-deficient mice (36).
However, Hougee et al (17) have demonstrated that probiotics could not stimulate the secretion of IL-10, but rather caused a reduction. Results from the present study support these latter observations. In the present study, the serum level of IL-10 was 
A

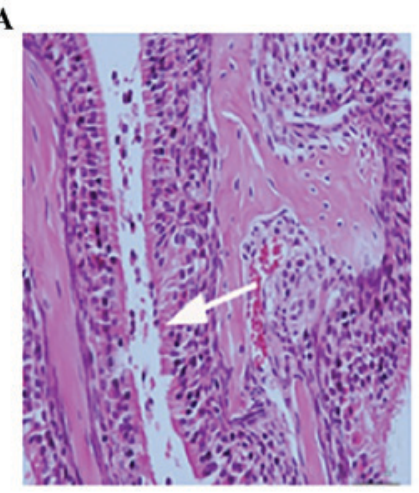

D

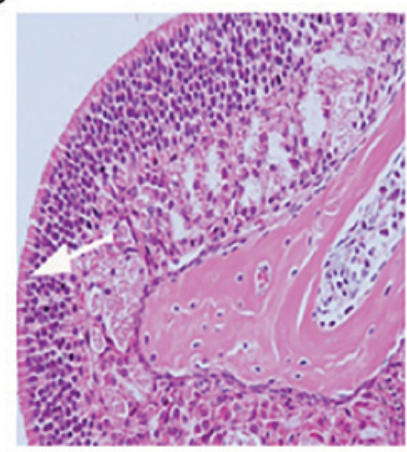

B

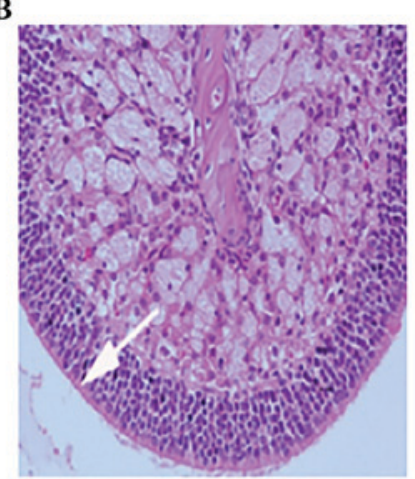

$\mathbf{E}$

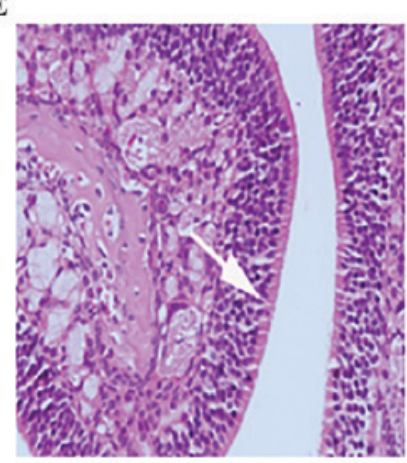

C

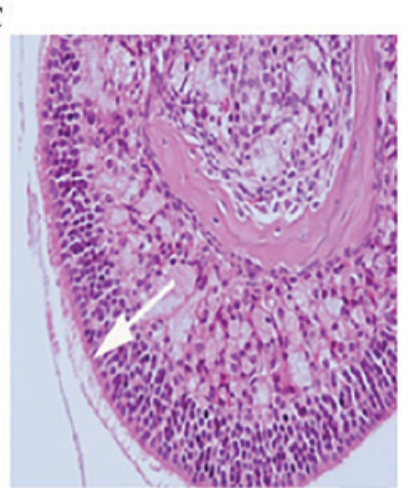

$\mathbf{F}$

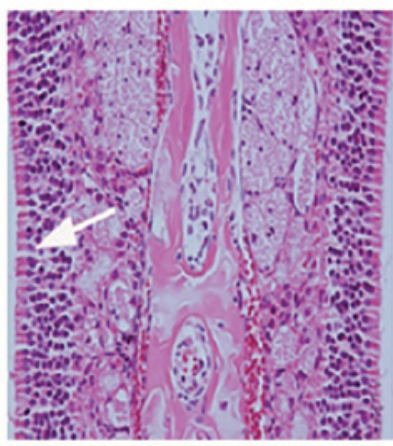

Figure 6. Nasal mucosa morphology of ovalbumin-sensitized mice following repeated administration of different doses of $B$. breve. (A) Positive control group; (B) negative control group; (C) $10^{10}$, (D) $10^{9}$, (E) $10^{7}$ and (F) $10^{5}$ CFU-treated groups (original magnification, x200; arrows indicate goblet cells). The nasal mucosal epithelial was intact following treatment with $B$. breve at doses of $10^{10}, 10^{9}$ and $10^{7} \mathrm{CFU}$, and the nasal mucosal congestion was reduced, compared with the positive control group; however, the 105 group did not markedly alleviate edema of the nasal mucosal tissue.

significantly lower in the $B$. breve treatment groups than that in the positive control group. It has been hypothesized that this decrease in free IL-10 in serum may be caused by an increase of IL-10 receptors (37). This is consistent with the findings of Schabussova et al (38) who demonstrated that the level of IL-10 mRNA was increased despite the decrease in serum IL-10 following probiotic administration.

It was demonstrated that even when AR mice were treated with a low dose $\left(10^{5} \mathrm{CFU}\right)$ of $B$. breve, the serum OVA-specific IgE level was significantly lower in comparison with the positive control group, indicating that even a low dose has an immunoregulatory effect. Notably, it was demonstrated that in the $10^{5}$ group, IL-10 was reduced and the proportion of CD4+CD25+ Tregs increased, whereas the level of the Th2 cytokine IL-4 did not change. This further supports that a low dose of $B$. breve may have an anti-inflammatory role depending on induction of Treg activity. The immunomodulatory effects of probiotics have been demonstrated in in vitro and clinical studies using low doses, whereas negative effects have been demonstrated at high doses (39). In the present study, the sneezing frequency in OVA-sensitized mice were significantly reduced in the higher dose groups, combined with decreased IL-4 and OVA-specific IgE levels and increased CD4+CD25+ Tregs in the spleen. In the low dose group, however, probiotics only decreased the levels of OVA-specific IgE and IL-10, and improved splenic CD4+CD25+ Treg levels, whereas there were no significant differences in sneezing frequency, nasal mucosa inflammation and IL-4 levels compared with the positive control group. However, a previous study by the present author (40), which analyzed the effect of different delivery modes (vaginal delivery vs. cesarean delivery) on immune status and nasal symptoms in AR mouse model, presented no significant differences in IL-4, IL-5 and IFN- $\gamma$ in serum levels. It is suggested that the contradictory results between these two studies may be mainly due to the doses, delivery modes or other mechanism, which requires more in-depth research. Therefore, it is speculated that a low dose of $B$. breve only enhances the function of CD4+CD25+ Tregs and does not increase Th1 response and/or weaken Th2 response. Previous clinical studies (41-43) have demonstrated that probiotics have the potential to improve the quality of life of patients with AR. Their nasal symptoms improved significantly following treatment with oral probiotics. However, the mechanism remains to be elucidated, and further studies should be conducted to explore its therapeutic effects and side effects.

A limitation of the present study is the lack of intestinal immune experiments; experiments regarding the effects of $B$. breve treatment in the composition of immune cells in the guts mucosa were not performed. It was intended to investigate fecal samples to explore the role of $B$. breve in the process of intestinal colonization and intestinal immune system. However, fecal samples were easily contaminated, which may have affected the veracity of results. Future studies should explore the association between intestinal colonization of microbes and AR.

Oral administration of $B$. breve has an anti-allergic effect by inhibiting the Th2 response and inducing the activity of CD4+CD25+ Tregs without evoking the Th1 response. Administration of $B$. breve at higher doses significantly reduced sneezing frequency, combined with decreased serum IL-4 and 
OVA-specific IgE levels and increased CD4+CD25+ Tregs in the spleen, and nasal mucosal epithelium was protected from local allergic reaction. However, a lower dose of $B$. breve could only slightly relieve the allergic reaction. The current study aided investigating the role of $B$. breve in immune response and treatment of AR and provided laboratory animal-based evidence, which may guide probiotic therapies and identifying the optimal dose of $B$. breve administration.

\section{Acknowledgements}

Not applicable.

\section{Funding}

The present study was supported by the National Natural Science Foundation of China (grant nos. 81170898 and 30801280), the International Scientific and Technological Cooperation Projects of Sichuan Province (grant no. 2016HH0064), the Project of Health and Family Planning Commission (grant no. 150113) to $\mathrm{YZ}$ and collaboration between Ghent University (Ghent, Belgium) and Sichuan University (Chengdu, China).

\section{Availability of data and materials}

All data generated or analyzed during this study are included in this published article.

\section{Authors' contributions}

JR and SH were the principal writers of the manuscript. JR, SH, YZha, DL, FY, CB, NZ, YZhe, JZ, SL and LL designed this study. JR, SH, FY, DL, JZ, YZhe and LL performed animal experiments and collected the preliminary data. YZha, $\mathrm{CB}$ and NZ performed English interpretation and correction of the manuscript. DL, JR, YZha, YZhe and JZ performed the statistical analysis and image production.

\section{Ethics approval and consent to participate}

All animal experiments were performed according to the Guide for the Care and Use of Laboratory Animals and approved by the Laboratory Animal Ethics Committee of Sichuan University (Chengdu, China).

\section{Patient consent for publication}

Not applicable.

\section{Competing interests}

The authors declare that they have no competing interests.

\section{References}

1. Rosenwasser LJ: Mechanisms of IgE Inflammation. Curr Allergy Asthma Rep 11: 178-183, 2011.

2. Pawankar R, Mori S, Ozu C and Kimura S: Overview on the pathomechanisms of allergic rhinitis. Asia Pac Allergy 1: 157-167, 2011.
3. Takahashi N, Kitazawa H, Iwabuchi N, Xiao JZ, Miyaji K, Iwatsuki K and Saito T: Immunostimulatory oligodeoxynucleotide from Bifidobacterium longum suppresses Th2 immune responses in a murine model. Clin Exp Immunol 145: 130-138, 2006.

4. Ashour HM and Niederkorn JY: Expansion of B cells is necessary for the induction of T-cell tolerance elicited through the anterior chamber of the eye. Int Arch Allergy Immunol 144: 343-346, 2007.

5. Ashour HM and Seif TM: The role of B cells in the induction of peripheral T cell tolerance. J Leukoc Biol 82: 1033-1039, 2007.

6. Kwon HK, Lee CG, So JS, Chae CS, Hwang JS, Sahoo A, Nam JH, Rhee JH, Hwang KC and Im SH: Generation of regulatory dendritic cells and CD4+ Foxp3+ T cells by probiotics administration suppresses immune disorders. Proc Natl Acad Sci USA 107: 2159-2164, 2010.

7. Jang SO, Kim HJ, Kim YJ, Kang MJ, Kwon JW, Seo JH, Kim HY, Kim BJ, Yu J and Hong SJ: Asthma prevention by Lactobacillus rhamnosus in a mouse model is associated with CD4(+)CD25(+) Foxp3(+) T cells. Allergy Asthma Immunol Res 4: 150-156, 2012.

8. Zhang LL, Chen X, Zheng PY, Luo Y, Lu GF, Liu ZQ, Huang H and Yang PC: Oral Bifidobacterium modulates intestinal immune inflammation in mice with food allergy. J Gastroenterol Hepatol 25: 928-934, 2010.

9. Jutel M, Akdis M, Budak F, Aebischer-Casaulta C, Wrzyszcz M, Blaser K and Akdis CA: IL-10 and TGF-beta cooperate in the regulatory $\mathrm{T}$ cell response to mucosal allergens in normal immunity and specific immunotherapy. Eur J Immunol 33: 1205-1214, 2003.

10. McFarland LV: Evidence-based review of probiotics for antibiotic-associated diarrhea and Clostridium difficile infections. Anaerobe 15: 274-280, 2009.

11. Caramia G: Gastroenteric pathology and probiotics: From myth to scientific evidence. Current aspects. Minerva Gastroenterol Dietol 55: 237-272, 2009 (In English, Italian).

12. Round JL and Mazmanian SK: The gut microbiota shapes intestinal immune responses during health and disease. Nat Rev Immunol 9: 313-323, 2009.

13. Kim HJ, Kim YJ, Lee SH, Kang MJ, Yu HS, Jung YH, Lee E, Seo JH, Kwon JW, Kim BJ, et al: Effects of Lactobacillus rhamnosus on asthma with an adoptive transfer of dendritic cells in mice. J Appl Microbiol 115: 872-879, 2013.

14. Castellazzi AM, Valsecchi C, Caimmi S, Licari A, Marseglia A, Leoni MC, Caimmi D, Miraglia del Giudice M, Leonardi S, La Rosa M and Marseglia GL: Probiotics and food allergy. Ital J Pediatr 39: 47, 2013.

15. Michail S: The role of probiotics in allergic diseases. Allergy Asthma Clin Immunol 5: 5, 2009.

16. Borchers AT, Selmi C, Meyers FJ, Keen CL and Gershwin ME: Probiotics and immunity. J Gastroenterol 44: 26-46, 2009.

17. Hougee S, Vriesema AJ, Wijering SC, Knippels LM, Folkerts G, Nijkamp FP, Knol J and Garssen J: Oral treatment with probiotics reduces allergic symptoms in ovalbumin-sensitized mice: A bacterial strain comparative study. Int Arch Allergy Immunol 151: 107-117, 2009.

18. Inoue $\mathrm{Y}$, Iwabuchi N, Xiao JZ, Yaeshima $\mathrm{T}$ and Iwatsuki $\mathrm{K}$ : Suppressive effects of Bifidobacterium breve strain M-16V on T-helper type 2 immune responses in a murine model. Biol Pharm Bull 32: 760-763, 2009.

19. Zhang L, Li N, Caicedo R and Neu J: Alive and dead Lactobacillus rhamnosus GG decrease tumor necrosis factor-alpha-induced interleukin-8 production in Caco-2 cells. J Nutr 135: 1752-1756, 2005.

20. Gill HS, Rutherfurd KJ, Cross ML and Gopal PK: Enhancement of immunity in the elderly by dietary supplementation with the probiotic Bifidobacterium lactis $\mathrm{HN} 019$. Am J Clin Nutr 74: 833-839, 2001.

21. Bohr UR, Selgrad M, Ochmann C, Backert S, König W, Fenske A, Wex T and Malfertheiner P: Prevalence and spread of enterohepatic Helicobacter species in mice reared in a specific-pathogen-free animal facility. J Clin Microbiol 44: 738-742, 2006.

22. National Research Council: Guide for the care and use of laboratory animals. 8th edition. National Academy Press, Washington, DC, 2011 .

23. Kawase M, He F, Kubota A, Harata G and Hiramatsu M: Orally administrated Lactobacillus gasseri TMC0356 and Lactobacillus GG alleviated nasal blockage of guinea pig with allergic rhinitis. Microbiol Immunol 51: 1109-1114, 2007.

24. Vliagoftis H, Kouranos VD, Betsi GI and Falagas ME: Probiotics for the treatment of allergic rhinitis and asthma: Systematic review of randomized controlled trials. Ann Allergy Asthma Immunol 101: 570-579, 2008. 
25. Fallon PG, Jolin HE, Smith P, Emson CL, Townsend MJ, Fallon R, Smith P and McKenzie AN: IL-4 induces characteristic Th2 responses even in the combined absence of IL-5, IL-9, and IL-13. Immunity 17: 7-17, 2002.

26. McLeod JJ, Baker B and Ryan JJ: Mast cell production and response to IL-4 and IL-13. Cytokine 75: 57-61, 2015.

27. Toru H, Pawankar R, Ra C, Yata J and Nakahata T: Human mast cells produce IL-13 by high-affinity IgE receptor cross-linking: Enhanced IL-13 production by IL-4-primed human mast cells. J Allergy Clin Immunol 102: 491-502, 1998.

28. Cameron L, Hamid Q, Wright E, Nakamura Y, Christodoulopoulos P, Muro S, Frenkiel S, Lavigne F, Durham S and Gould $\mathrm{H}$ : Local synthesis of epsilon germline gene transcripts, IL-4, and IL-13 in allergic nasal mucosa after ex vivo allergen exposure. J Allergy Clin Immunol 106: 46-52, 2000.

29. Chung F: Anti-inflammatory cytokines in asthma and allergy: Interleukin-10, interleukin-12, interferon-gamma. Mediators Inflamm 10: 51-59, 2001.

30. Tang C, Inman MD, van Rooijen N, Yang P, Shen H, Matsumoto K and O'Byrne PM: Th type 1-stimulating activity of lung macrophages inhibits Th2-mediated allergic airway inflammation by an IFN-gamma-dependent mechanism. J Immunol 166: 1471-1481, 2001

31. Ghadimi D, Fölster-Holst R, de Vrese M, Winkler P, Heller KJ and Schrezenmeir J: Effects of probiotic bacteria and their genomic DNA on TH1/TH2-cytokine production by peripheral blood mononuclear cells (PBMCs) of healthy and allergic subjects. Immunobiology 213: 677-692, 2008.

32. Vuotto C, Longo F and Donelli G: Probiotics to counteract biofilm-associated infections: Promising and conflicting data. Int J Oral Sci 6: 189-194, 2014.

33. Ozdemir O: Various effects of different probiotic strains in allergic disorders: An update from laboratory and clinical data. Clin Exp Immunol 160: 295-304. 2010.

34. Sakaguchi S, Yamaguchi T, Nomura T and Ono M: Regulatory T cells and immune tolerance. Cell 133: 775-787, 2008.

35. Nouri-Aria KT and Durham SR: Regulatory T cells and allergic disease. Inflamm Allergy Drug Targets 7: 237-252, 2008.

36. Madsen K, Cornish A, Soper P, McKaigney C, Jijon H, Yachimec C, Doyle J, Jewell L and De Simone C: Probiotic bacteria enhance murine and human intestinal epithelial barrier function. Gastroenterology 121: 580-591, 2001.
37. Winkler B, Bolwig C, Seppälä U, Spangfort MD, Ebner C and Wiedermann U: Allergen-specific immunosuppression by mucosal treatment with recombinant Ves $\mathrm{v} 5$, a major allergen of Vespula vulgaris venom, in a murine model of wasp venom allergy. Immunology 110: 376-385, 2003.

38. Schabussova I, Hufnagl K, Wild C, Nutten S, Zuercher AW, Mercenier A and Wiedermann U: Distinctive anti-allergy properties of two probiotic bacterial strains in a mouse model of allergic poly-sensitization. Vaccine 29: 1981-1990, 2011.

39. Smits HH, Engering A, van der Kleij D, de Jong EC, Schipper K, van Capel TM, Zaat BA, Yazdanbakhsh M, Wierenga EA, van Kooyk Y and Kapsenberg ML: Selective probiotic bacteria induce IL-10-producing regulatory $\mathrm{T}$ cells in vitro by modulating dendritic cell function through dendritic cell-specific intercellular adhesion molecule 3-grabbing nonintegrin. J Allergy Clin Immunol 115: 1260-1267, 2005.

40. Ren JJ, Yu Z, Yang FL, Lv D, Hung S, Zhang J, Lin P, Liu SX, Zhang $\mathrm{N}$ and Bachert $\mathrm{C}$ : Effects of bifidobacterium breve feeding strategy and delivery modes on experimental allergic rhinitis mice. PLoS One 10: e0140018, 2015.

41. Singh A, Hacini-Rachinel F, Gosoniu ML, Bourdeau T, Holvoet S, Doucet-Ladeveze R, Beaumont M, Mercenier A and Nutten S: Immune-modulatory effect of probiotic Bifidobacterium lactis NCC2818 in individuals suffering from seasonal allergic rhinitis to grass pollen: An exploratory, randomized, placebo-controlled clinical trial. Eur J Clin Nutr 67: 161-167, 2013.

42. Dennis-Wall JC, Culpepper T, Nieves C Jr, Rowe CC, Burns AM, Rusch CT, Federico A, Ukhanova M, Waugh S, Mai V, et al: Probiotics ((Lactobacillus gasseri KS-13, Bifidobacterium bifidum G9-1, and Bifidobacterium longum MM-2) improve rhinoconjunctivitis-specific quality of life in individuals with seasonal allergies: A double-blind, placebo-controlled, randomized trial. Am J Clin Nutr 105: 758-767, 2017.

43. Miraglia Del Giudice M, Indolfi C, Capasso M, Maiello N, Decimo F and Ciprandi G: Bifidobacterium mixture (B longum BB536, B infantis M-63, B breve M-16V) treatment in children with seasonal allergic rhinitis and intermittent asthma. Ital J Pediatr 43: 25, 2017.

This work is licensed under a Creative Commons Attribution-NonCommercial-NoDerivatives 4.0 International (CC BY-NC-ND 4.0) License. 